\title{
A cell-centred CVD-MPFA finite-volume method for two-phase fluid flow problems with capillary heterogeneity and discontinuity
}

\author{
Raheel Ahmed • Yawei Xie • Michael G. Edwards
}

October 7, 2018

\begin{abstract}
A novel finite-volume method is presented that is applicable to discontinuous capillary pressure fields. The method is developed within the control-volume distributed multi-point flux approximation (CVD-MPFA) framework (Edwards and Rogers, 1998; Friis et al, 2008). Results are computed on structured and unstructured grids that demonstrate the ability of the method to resolve flow in the presence of a discontinuous capillary pressure field for diagonal and full-tensor permeability fields. In addition to an upwind approximation for the saturation equation flux, the importance of upwinding on capillary pressure flux via a hybrid formulation is shown.
\end{abstract}

Keywords Finite-volume $\cdot$ CVD-MPFA · two-phase $\cdot$ capillary pressure $\cdot$ heterogeneity $\cdot$ discontinuity threshold pressure

\section{Introduction}

In this work, a new control-volume distributed multi-point flux approximation (CVD-MPFA) finite volume method is developed for two-phase flow with a possibly heterogeneous and discontinuous capillary pressure field, building on the framework of (Edwards and Rogers, 1998, Friis et al, 2008). The CVD-MPFA finite

5 volume formulation is an optimal approximation when compared to other methods, in that this formulation provides a flux-continuous locally conservative Darcy-flux approximation that is consistent for flow problems involving full-tensor permeability fields on structured and unstructured grids, while only depending on a single degree of freedom (unknown) per flow variable per control-volume. These properties provide the motivation for this CVD-MPFA development. Immiscible two-phase incompressible flow is governed by 10 a system of equations which involve a non-linear convection-diffusion equation for the saturation and an elliptic partial-differential-equation for phase pressure with a capillary pressure term on the right hand side. Capillary pressure is the difference between non-wetting and wetting immiscible phase pressures across an interface, and varies as a function of saturation throughout the field. The capillary pressure has a rock property functional dependence on saturation defined by rock dependent capillary-saturation curves of the 5 porous medium. Each rock type, in the domain, has its own intrinsic rock properties, including local permeability variation and local capillary pressure curves, that vary as a function of saturation. Brooks-Corey and Van Genuchten are commonly used functions for capillary pressure (van Duijn and de Neef, 1998). Capillary heterogeneity refers to a geological description where the domain consists of different rock types that have different capillary-saturation curves. Varying rock properties can result in discontinuous capillary 20 pressure variation across neighbouring rock interfaces. Permeability heterogeneity, anisotropy, porosity and capillary heterogeneity can all have profound effects on local flow direction and behaviour. Preferential flow directions and flow rates typically occur in regions of high permeability contrast, while capillary forces can drastically change the nature of flow paths and cause the flow to spread and be more diffuse. Oil recovery may be significantly reduced due to the effects of capillary heterogeneity, a phenomenon commonly referred

Raheel AHMED ${ }^{1}$

E-mail: raheel_ahmed05@live.com

${ }^{1}$ Dimue Technology Co, Ltd., Wuhan 430223, China

Yawei XIE ${ }^{2}$

E-mail: 717023@swansea.ac.uk

Michael G. EDwArds ${ }^{2}$

E-mail: m.g.edwards@swansea.ac.uk

2 ZCCE, College of Engineering, Swansea University, Swansea SA18EN, Wales UK 
to, as oil trapping (van Duijn et al, 1995, Brenner et al, 2013). Adjacent rocks may have different capillarysaturation curves, visual examples of capillary-saturation curves are given in Fig. 1c and Fig. 1d, For the maximum saturation $s=1.0$, the capillary pressure is known as the threshold pressure $\phi_{t}$ (also known as entry pressure). The difference in the threshold capillary pressures of adjacent rocks decides continuity or discontinuity of capillary pressures between the rocks.

30 Because of the importance of the capillary discontinuity, many authors have been working on this topic using various numerical methods. For example standard Galerkin and Petrov-Galerkin have been used by Helmig and Huber (1998). Hoteit and Firoozabadi (2008) presented the mixed finite-element method for the solution of discontinuous capillary pressure. The discontinuous-Galerkin method, presented in (Bastian, 2014), deals with discontinuous capillary pressure and numerical simulations are presented. The convergence

35 of finite-volume schemes accounting for discontinuous capillary pressure have been analysed in (Enchéry et al, 2006, Cancès, 2009). A cell-centred finite-volume scheme with two-point flux approximation (TPFA) has been studied in (van Duijn et al, 1995, Brenner et al, 2013) in the context of discontinuous capillary pressure. A cell-centred finite-volume method with multi-point flux approximation (L-method) is formulated in (Wolff et al, 2013) for two-phase flow, however we have not been able to find a complete formulation

40 with description that includes discontinuous capillary pressure. Vertex-centred finite-volume schemes for capillary heterogeneities are presented in (Niessner et al, 2005, Papafotiou et al, 2010). More recent related works include (Arbogast et al, 2013; Moortgat and Firoozabadi, 2013; Li and Tchelepi, 2015, Brenner et al, 2016, Hamon et al, 2018).

Here, we extend the cell-centred multi-point flux approximation (CVD-MPFA) with triangular pressure sup45 port (Friis et al, 2008) to approximate the capillary pressure flux while taking account of any discontinuity in capillary pressure.

\section{Two-phase flow model}

Two-phase flow consists of a wetting-phase and a non-wetting phase, that we assume here are water and oil respectively. The flow equations are written in the $V a$ fractional flow form (Hoteit and Firoozabadi, 2008)

in this work with the aqueous phase continuity equation written as:

$$
\varphi \frac{\partial s}{\partial t}+\nabla \cdot\left(f_{w} \boldsymbol{v}_{a}\right)=q_{w}
$$

where $s=s_{w}(\boldsymbol{x}, t)$ is the water saturation, $f_{w}(s)=\lambda_{w} / \Lambda$ is the aqueous fractional flow which involves the ratio of water mobility $\lambda_{w}$ to the total mobility $\Lambda=\lambda_{w}+\lambda_{o}$. The rock permeability tensor, denoted by $\boldsymbol{k}$, is heterogeneous spatially varying and second rank in two-dimensions while $\phi_{c}, \varphi$ and $q_{w}$ are the capillary pressure, porosity and the known aqueous source term respectively. Capillary pressure is the

55 difference between the non-wetting and wetting phase pressures i.e., $\phi_{c}=\phi_{o}-\phi_{w}$. In this work gravity is omitted. Oil saturation $s_{o}$ is deduced from the volume balance $s_{w}+s_{o}=1$. The velocity $\boldsymbol{v}_{a}$ is defined as $\boldsymbol{v}_{a}=-\Lambda \boldsymbol{K} \nabla \phi$ where $\phi=\phi_{w}$ is the aqueous phase pressure which is obtained by solving the pressure equation:

$$
-\nabla \cdot(\Lambda K \nabla \phi)=\nabla \cdot\left(\lambda_{o} \boldsymbol{K} \nabla \phi_{c}\right)+q_{w}+q_{o}
$$

which results from mass conservation and Darcy's law, and for incompressible flow describes a divergence ${ }_{0}$ free total velocity away from any (well) source, where $\boldsymbol{v}_{t}=\boldsymbol{v}_{a}+\boldsymbol{v}_{c}$ and $\boldsymbol{v}_{c}=-\lambda_{o} \boldsymbol{K} \nabla \phi_{c}$. In this work, the CVD-MPFA finite volume method (Edwards and Rogers, 1998, Friis et al, 2008) is developed to approximate capillary pressure flux and compute the solution of the resulting phase pressure equation (2). The implicitpressure explicit-saturation (IMPES) method is employed for the solution of the coupled system of equations (1) and (2) (Aziz and Settari, 1986). Here, a cell-centred first order upwind (Aziz and Settari, 1986) finite65 volume method is used for approximation of the convective flux of the saturation equation (1).

The $V a$ formulation proposed in (Hoteit and Firoozabadi, 2008, Friis and Evje, 2012, Bastian, 2014) only involves explicit capillary flux discretisation on the right hand side of the pressure equation. The key advantage of the $V a$ formulation is that the capillary pressure does not appear explicitly in the saturation equation since the flux is proportional to $V a$ which depends on the gradient of the aqueous phase pressure,

70 which is an attractive simplification. The saturation equation employs an upwind flux with upwind direction defined by the local $\mathrm{Va}$ flux resolved on each control-volume facet, and with stability of the explicit update of the saturation equation governed by a CFL condition based on the $V a$ flux. In effect the $V a$ flux is comprised of a convection-diffusion flux, since capillary flux is indirectly embedded in the operator, which is only seen when expressing $V a$ in terms of $V_{t}$ (see above), consequently the CFL is more restrictive due

75 to being based effectively on an artificial wave speed. We note that a $V_{t}$ formulation using the actual wave speed together with the non-linear diffusive flux resulting from capillary pressure is presented in (Xie and Edwards, 2017). Consistent with upwinding on $V a$, then the capillary flux in the divergence term on the 
right hand side of the pressure equation, is treated as an artificial wave speed, and the capillary flux oil-phase mobility is upwinded according to the sign of the capillary flux resolved on each control-volume facet, which ${ }_{80}$ is demonstrated (results section) to aid stability when capillary pressure is discontinuous. An analogous upwind approximation was proposed by (Hoteit and Firoozabadi, 2008). An example of the contrast between using a centred approximation and the upwind approximation for capillary flux oil-phase mobility is also presented in the results section. Instability of an explicit forward time centred approximation of convective flux is well known e.g. LeVeque (1992), and instability resulting from using the centred oil-phase mobility approximation is clearly seen c.f. section 6.5. in contrast to the stable upwind approximation results. The two types of upwinding used here on $V a$ and capillary flux respectively, create a hybrid $V a$ formulation. We note that if gravity is present the $V a$ formulation is only valid for convective dominant flow regimes (Xie and Edwards, 2017). However since gravity is not considered here we use the above hybrid $V a$ formulation.

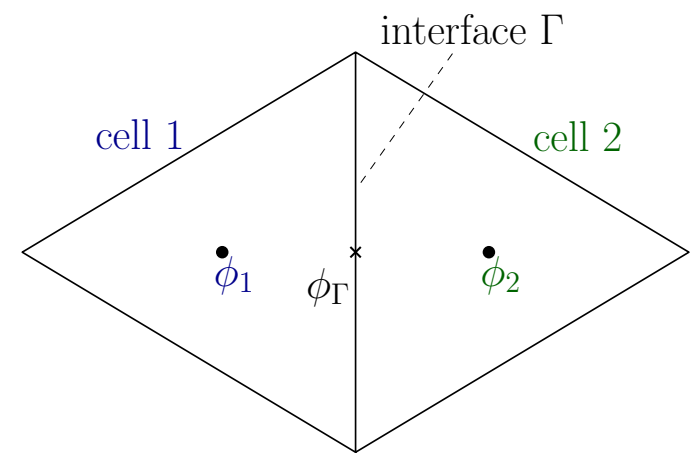

(a) Phase-pressure continuity on the interface between adjacent cells

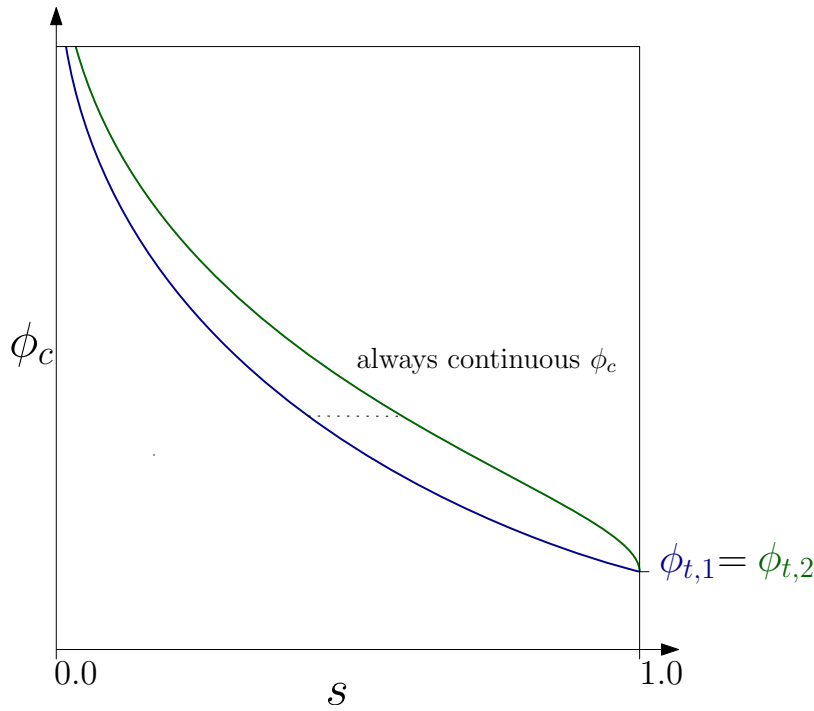

(c) Capillary-saturation curves for two rocks with equal threshold capillary pressures

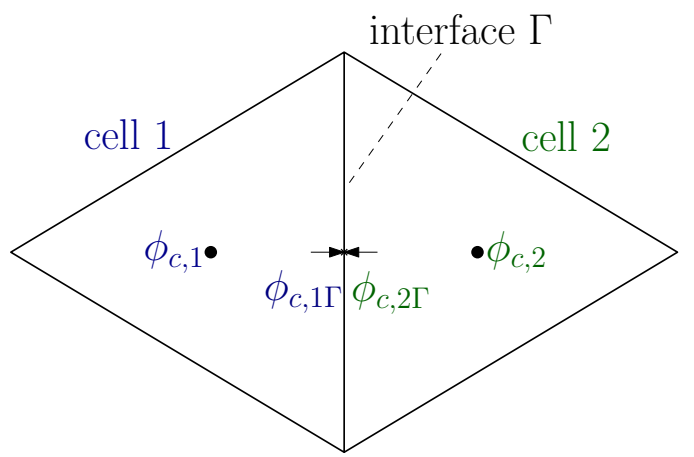

(b) Discontinuous capillary pressure on the interface between adjacent cells

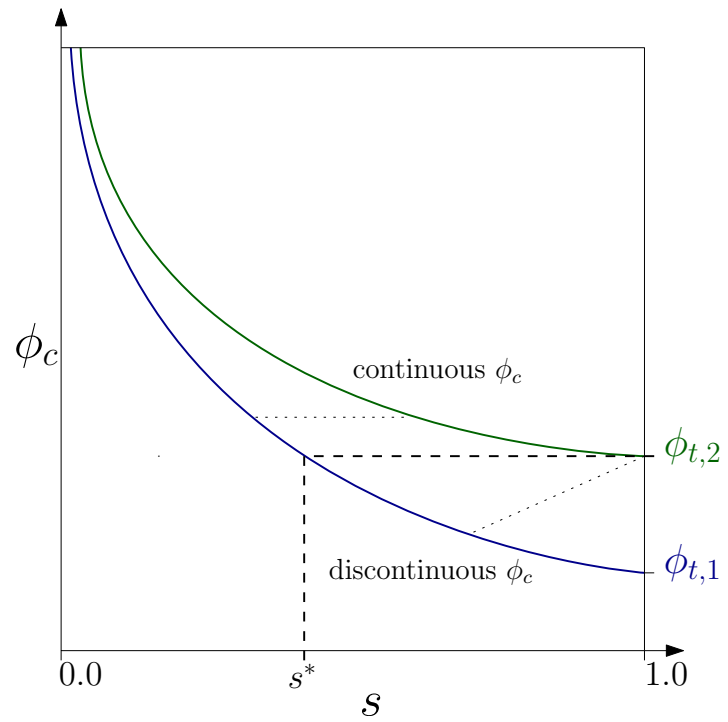

(d) Capillary-saturation curves for two rocks with different threshold capillary pressures

Fig. 1: Capillary heterogeneity across two rocks

\section{Capillary interface conditions}

Two essential conditions of the CVD-MPFA method are (i) pressure continuity and (ii) flux-continuity (Edwards and Rogers, 1998, Friis et al, 2008). When fluxes are approximated using the phase pressure $\phi$ (left side of Eq. (2)), the phase pressure is always considered to be continuous on the interface between two cells as shown in Fig. 1a. Thus interface pressure can be eliminated by the usual CVD-MPFA fluxcontinuity conditions. But, when the fluxes involving capillary pressure are approximated (right side of Eq. (2), account must be taken of a possible discontinuous capillary pressure on the interface between two 
cells as shown in Fig. 1b The discontinuity of the capillary pressure depends on the difference between the threshold pressure of the adjacent cells that are associated with the different rock types. Consider two cells, Fig. 1b that belong to two different rock types 1 and 2 having the threshold pressures $\phi_{t, 1}$ and $\phi_{t, 2}$, respectively. We consider $\phi_{t, 1} \leq \phi_{t, 2}$. The possible discontinuous capillary pressures on the sides of the interface are denoted by $\phi_{c, 1 \Gamma}$ and $\phi_{c, 2 \Gamma}$ corresponding to cells 1 and 2 , respectively. We have two conditions for the approximation of capillary pressure flux on the two interface sides, following van Duijn et al, 1995, Bastian, 2014). The first condition is the essential flux-continuity condition, with analogous treatment to that for the phase-pressure. The second condition is the extended pressure condition (van Duijn et al, 1995) written as:

$$
\begin{array}{llr}
\text { If } \phi_{c, 1 \Gamma} \geqslant \phi_{t, 2} & \rightarrow \text { continuity: } \begin{array}{r}
\phi_{c, 1 \Gamma}=\phi_{c, 2 \Gamma}=\phi_{c, \Gamma} ; \\
\phi_{c, \Gamma} \text { is eliminated using flux-continuity }
\end{array} \\
\text { or } \quad \rightarrow \text { discontinuity: } & \phi_{c, 2 \Gamma}=\phi_{t, 2} ;
\end{array}
$$

or

If condition (3) is satisfied on the interface then we employ continuity of capillary pressure, otherwise the capillary pressure is discontinuous. When the capillary-saturation curves of the adjacent rocks are such that the threshold pressures are equal as shown in Fig. 1c the condition (3) is always satisfied for all values of saturation implying that the capillary pressure is always continuous in this case. The resulting 9 continuous capillary pressure is eliminated by using the flux-continuity condition analogous to the case of phase-pressure. Now, consider a case when the adjacent rocks (e.g. 1 and 2 ) have capillary-saturation curves such that the threshold pressures are different $\left(\phi_{t, 1}<\phi_{t, 2}\right)$ as shown in the Fig. $1 \mathrm{~d}$ corresponding to the cells in Fig. $1 \mathrm{~b}$. There is a threshold saturation $s_{w}^{*}$ for cell 1 such that the corresponding capillary pressure is equal to the threshold pressure of cell 2 i.e. $\phi_{c, 1}\left(s^{*}\right)=\phi_{t, 2}$. Whenever the saturation in cell 1 is lower than the threshold saturation i.e. $s_{1} \leq s^{*}$ (the corresponding capillary pressure on the interface in cell 1 is higher than the threshold pressure of cell 2 i.e. $\phi_{c, 1} \geq \phi_{t, 2}$ ), we have the condition (3) satisfied and the capillary pressure across the interface is continuous. The continuous capillary pressure on the interface is eliminated by using the condition of continuity of flux across the interface. Now, if the saturation in cell 1 is higher than the threshold saturation i.e. $s_{1}>s^{*}$ (the corresponding capillary pressure on the interface in cell 1 is lower than the threshold pressure of cell 2 i.e. $\phi_{c, 1}<\phi_{t, 2}$ ), we cannot satisfy the condition (3), so the capillary pressure on the cell 2 interface is imposed with the value equal to the threshold pressure i.e. $\phi_{c, 2 \Gamma}=\phi_{t, 2}$ and we eliminate $\phi_{c, 1 \Gamma}$ by using continuity of flux across the interface. In this way, we treat a discontinuity in capillary pressure which depends on the saturation (and capillary pressure) across the interface between the two cells.

\section{CVD-MPFA phase-flux and capillary-flux approximation}

We approximate the pressure equation (2) using the finite volume CVD-MPFA method. The pressure equation (2) is integrated over each grid cell control volume using the Gauss divergence theorem to obtain:

$$
-\int_{\partial \Omega_{i}}(\Lambda \boldsymbol{k} \nabla \phi) \cdot \boldsymbol{n}_{\boldsymbol{i}} d S=\int_{\partial \Omega_{i}}\left(\lambda_{o} \boldsymbol{k} \nabla \phi_{c}\right) \cdot \boldsymbol{n}_{\boldsymbol{i}} d S+\int_{\Omega_{i}} q_{w} d V+\int_{\Omega_{i}} q_{o} d V
$$

The discrete approximation of equation (5) is written in terms of the summation of the fluxes on all the

half-edges of the grid-cells:

$$
\sum_{i}^{2 N_{e}} \Lambda_{i} F_{i}=\sum_{i}^{2 N_{e}} \lambda_{o i} F_{c i}+\bar{q}
$$

where, $N_{e}$ is the total number of edges of the grid-cell $\left(N_{e}=3\right.$ for the triangle cell), $\bar{q}=\int_{\Omega_{i}} q_{w} d V+\int_{\Omega_{i}} q_{o} d V$. $F_{i}$ and $F_{c i}$ are outward normal fluxes on a half-edge $i$ of the grid-cell corresponding to the phase-pressure and the capillary pressure, respectively. The fluxes $F_{i}$ and $F_{c i}$ are approximated in terms of the respective cell-centred phase and capillary pressures using the CVD-MPFA formulation (Friis et al, 2008). Details of the new capillary flux approximation are given in the next subsection below. Here, we employ the symmetric positive definite (SPD) triangular pressure support CVD-MPFA scheme (Friis et al, 2008).

We now outline the flux construction. We work on a cluster of cells common to a given vertex and approximate the fluxes on all the cell half-edges common to that vertex following (Friis et al, 2008). Consider a cluster of three cells common to the vertex $V$ as shown in Fig. 2a. For the phase pressure fluxes, we introduce auxiliary pressures on the interfaces, as shown in Fig. 2c and the phase pressure is continuous across the interfaces. Interface pressures and flux continuity quadrature points are defined by the parametric 
quadrature variation $[0<q \leq 1]$ along each sub-interface, and for the SPD scheme $q=2 / 3$ which defines quadrature points positioned at $1 / 3$ of the length of each triangle cell edge, Fig. 2c shows $\phi_{A}, \phi_{B}, \phi_{C}$ located at the SPD quadrature points of the cluster. Triangle pressure support (TPS) is used where pressure has a piecewise linear variation over each sub-cell triangle, e.g. over the triangle sub-cell 1 pressure is written in terms of barycentric coordinates $(\xi, \eta)$ where:

$$
\phi=(1-\xi-\eta) \phi_{1}+\xi \phi_{A}+\eta \phi_{C}
$$

A piecewise constant pressure gradient vector is formed over each sub-cell from the pressure field, from which the Darcy velocity vector can be determined in each sub-cell. The Darcy velocity is resolved along the two outward sub-interface normals of the sub-cell. The normal flux at the left hand side of sub-interface $I_{A}$ is given by velocity resolution along the normal vector $\boldsymbol{d} \boldsymbol{L}_{\boldsymbol{h}}=0.5\left(\left(y_{V}-y_{V_{A}}\right),-\left(x_{V}-x_{V_{A}}\right)\right)^{t r}$ outward normal to cell 1:

$$
F_{I_{A}}^{1}=\tilde{\boldsymbol{v}}_{\boldsymbol{a}} \cdot \boldsymbol{d} \boldsymbol{L}_{\boldsymbol{h}}=-\left.\left(T_{11}^{1} \phi_{\xi}+T_{12}^{1} \phi_{\eta}\right)\right|_{A} ^{1}
$$

where $\tilde{\boldsymbol{v}}_{\boldsymbol{a}}=-\boldsymbol{k} \nabla \phi, \boldsymbol{T}=\boldsymbol{T}(q)$ is a discrete approximation of the general Piola tensor (in physical space) and define the coefficients of $\left(\phi_{\xi}, \phi_{\eta}\right)^{t r}$. Further details of the general tensor approximation are given in

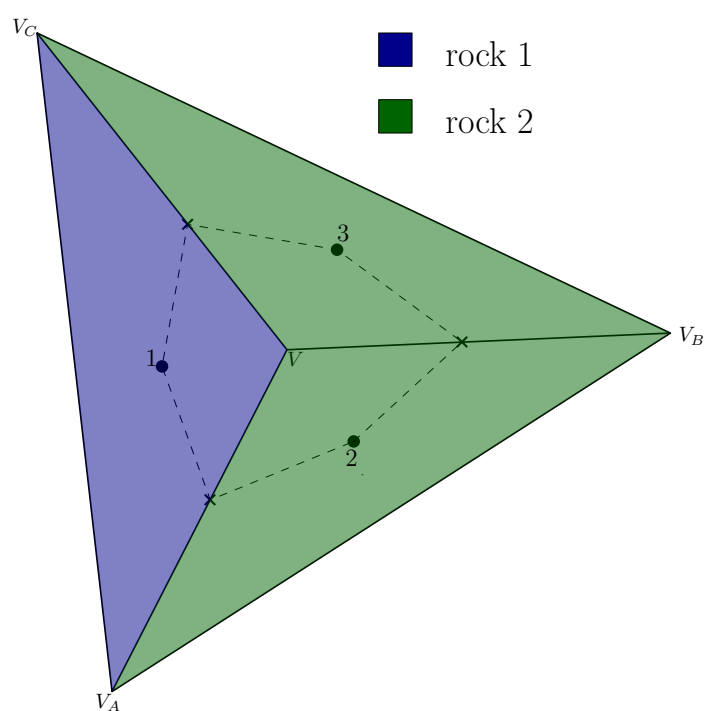

(a) Cluster of cells illustrating the rock-types of the cells.

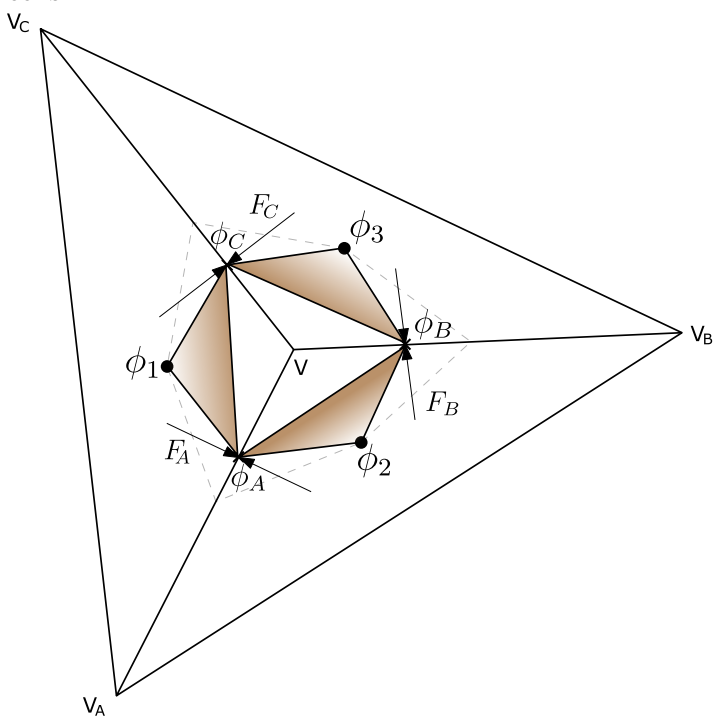

(c) Cluster of cells with illustration of fluxes, continuous phase-pressures with sub-cell triangular pressure support

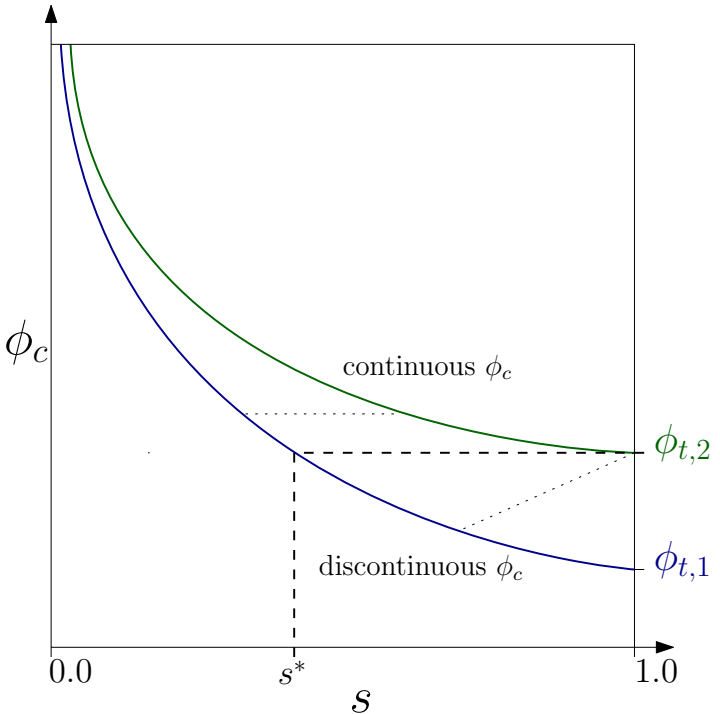

(b) Capillary-saturation curves for two rocks with different threshold capillary pressures

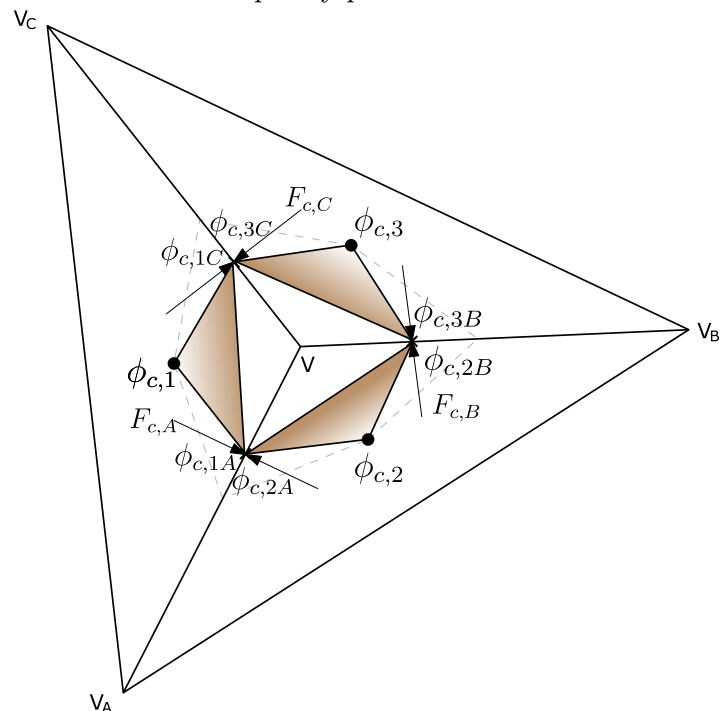

(d) Cluster of cells with illustration of fluxes, discontinuous capillary pressures with sub-cell triangular pressure support

Fig. 2: Cluster and dual-cell of three 2D cells belonging to two rock-types. 
(Friis et al, 2008). Similarly, fluxes are determined on other sub-interfaces as well. The system of continuous

fluxes on the left and right hand sides of the sub-interfaces (half edges) are written as;

$$
\boldsymbol{F}=\mathrm{A}_{\mathrm{L}} \Phi+\mathrm{B}_{\mathrm{L}} \Phi_{I}=\mathrm{A}_{\mathrm{R}} \Phi+\mathrm{B}_{\mathrm{R}} \Phi_{I}
$$

where flux continuity is imposed, and $\boldsymbol{F}=\left(F_{A}, F_{B}, F_{C}\right)^{t r}, \Phi=\left(\phi_{1}, \phi_{2}, \phi_{3}\right)^{t r}$ and $\Phi_{I}=\left(\phi_{A}, \phi_{B}, \phi_{C}\right)^{t r}$. Flux continuity is imposed on every sub-interface e.g, for $I_{A}$ flux continuity is written as,

$$
F_{I_{A}}^{1}=-F_{I_{A}}^{2}
$$

and expanded in tensor form

$$
-\left.\left(T_{11}^{1} \phi_{\xi}+T_{12}^{1} \phi_{\eta}\right)\right|_{A} ^{1}=\left.\left(T_{21}^{2} \phi_{\xi}+T_{22}^{2} \phi_{\eta}\right)\right|_{A} ^{2}
$$

The auxiliary interface pressures $\Phi_{I}$ are eliminated via the above flux continuity conditions to obtain: 145 $\boldsymbol{F}=\overline{\mathrm{A}} \Phi$, where $\overline{\mathrm{A}}$ denotes the resulting matrix after elimination of $\Phi_{I}$. The fluxes are then assembled in the discrete integrated divergence of Darcy velocity on the left hand side of Eq. (6).

\subsection{Capillary flux}

Now we describe the capillary pressure flux approximation. The right hand side of Eq. (6) involves the discrete divergence of capillary flux. Approximation of capillary flux is illustrated in Fig. 2d, which shows a cluster of capillary pressure sub-cell triangle basis functions. Similar to phase pressure, a TPS approximation of capillary pressure is employed, with a piecewise linear variation over each sub-cell triangle, e.g. over the triangle sub-cell 1 capillary pressure is written in terms of barycentric coordinates $(\xi, \eta)$ where:

$$
\phi_{c}=(1-\xi-\eta) \phi_{c, 1}+\xi \phi_{c, 1 A}+\eta \phi_{c, 1 C}
$$

and the normal flux at the left hand side of sub-interface $I_{A}$ is given by velocity resolution along the normal

$$
F_{c, I_{A}}^{1}=\tilde{\boldsymbol{v}}_{\boldsymbol{c}} \cdot \boldsymbol{d} \boldsymbol{L}_{\boldsymbol{h}}=-\left.\left(T_{11}^{1} \phi_{c, \xi}+T_{12}^{1} \phi_{c, \eta}\right)\right|_{A} ^{1}
$$

where $\tilde{\boldsymbol{v}}_{\boldsymbol{c}}=-\boldsymbol{k} \nabla \phi_{c}$. Similarly, fluxes are defined on the left and right hand sides of the sub-interfaces, however account must now be taken of the conditions ((3), (4)).

We check the conditions ((3), (4) ) across each of the interfaces by using the cell-centred values to decide on continuity or discontinuity of capillary pressure across the interfaces. The rock types of the cells are illustrated in Fig. 2a, which correspond to the saturation-capillary curves shown in Fig. 2b. Consider the situation for example, where $\phi_{c, 1}<\phi_{t, 2}$. In this case, we cannot satisfy condition $((3))$, as there is a discontinuity in the capillary pressure on both interfaces $A$ and $C$, which corresponds to the condition (44)), resulting in the imposition $\phi_{c, 2 A}=\phi_{t, 2}$ and $\phi_{c, 3 C}=\phi_{t, 2}$. As cells 2 and 3 belong to the same rock-types, the capillary pressure will always be continuous on the interface between these i.e. $\phi_{c, 2 B}=\phi_{c, 3 B}=\phi_{c, B}$. In this case, the system of continuous capillary fluxes on the left and right hand sides of the sub-interfaces (half edges) are written as:

$$
\boldsymbol{F}_{c}=\mathrm{A}_{c L} \Phi_{c}+\mathrm{B}_{c L} \Phi_{c, I}+\mathrm{C}_{c L} \Phi_{c, d i s}=\mathrm{A}_{c R} \Phi_{c}+\mathrm{B}_{c R} \Phi_{c, I}+\mathrm{C}_{c R} \Phi_{c, d i s}
$$

where, $\boldsymbol{F}_{c}=\left(F_{c, A}, F_{c, B}, F_{c, C}\right)^{t r}, \Phi_{c}=\left(\phi_{c, 1}, \phi_{c, 2}, \phi_{c, 3}\right)^{t r}, \Phi_{c, I}=\left(\phi_{c, 1 A}, \phi_{c, B}, \phi_{c, 1 C}\right)^{t r}$ and $\Phi_{c, d i s}=$ $\left(\phi_{c, 2 A}, \phi_{c, 3 C}\right)^{t r}$. As $\Phi_{c, d i s}\left(\phi_{c, 2 A}=\phi_{c, 3 C}=\phi_{t, 2}\right)$ and $\Phi_{c}$ has known numerical values, so these values are used in the approximation of the fluxes. The above flux continuity conditions are used on the interfaces to eliminate $\Phi_{c, I}$. Thus, we can express capillary pressure fluxes as $\boldsymbol{F}_{c}=\overline{\mathrm{A}}_{c} \Phi_{c}+\overline{\mathrm{C}}_{c} \Phi_{c, \text { dis }}$ which are then assembled to form the discrete divergence of capillary flux on the right hand side of Eq. (6). 


\section{Convective $V a$ flux approximation}

The convective flux approximation involves approximations of mobility and fractional flow that appear in the discrete pressure equation and saturation equation respectively. The discrete interface total mobility $\Lambda_{i}$ c.f. left hand side of the pressure equation $(6)$, is defined by the average of cell neighbour values, e.g. referring to interface $A$ of Fig. 2c then

$$
\Lambda_{A}=\frac{1}{2}\left(\Lambda\left(s_{1}\right)+\Lambda\left(s_{2}\right)\right) .
$$

As discussed above the interface oil-phase mobility c.f. right hand side of equation (6) is upwinded, here according to the sign of the local capillary phase flux resolved on each control-volume face, where e.g. referring to interface $A$ of Fig. $2 \mathrm{~d}$

$$
\lambda_{o A}= \begin{cases}\lambda_{o}\left(s_{1}\right) & \text { if } \quad\left(F_{c, A}\right) \geq 0, \\ \lambda_{o}\left(s_{2}\right) & \text { if }\left(F_{c, A}\right)<0 .\end{cases}
$$

where $F_{c, A}$ is defined above in section 4.1 which leads to a stable approximation. We note that the centred approximation of $\lambda_{o}$ with $\lambda_{o A}=\frac{1}{2}\left(\lambda_{o}\left(s_{1}\right)+\lambda_{o}\left(s_{2}\right)\right)$ leads to the unstable approximation discussed above, and a test case demonstrating the resulting instability is presented in the results section 6.5 .

The discrete saturation equation is written as

$$
\frac{\left(s_{i}^{n+1}-s_{i}^{n}\right)}{\Delta t} A_{i}=-\sum_{i}^{2 N_{e}} f_{w_{i}}^{n} \Lambda_{i}^{n} F_{i}+q_{w} A_{i}
$$

which employs explicit forward (Euler) time stepping, where $s_{i}^{n}$ is saturation at cell $i$ and time level $n, A_{i}$ is the cell area, $\Delta t$ is the time step and the total mobility flux $\Lambda_{i} F_{i}$ is defined above. Also as discussed above, the CFL is based on the $V a$ wave speed, and in the $V a$ formulation fractional flow $f_{w}$ is upwinded, here according to the sign of the local $\mathrm{Va}$ flux resolved on each control-volume face, where e.g. again referring to interface $A$ of Fig. $2 \mathrm{c}$

$$
f_{w_{A}}= \begin{cases}f_{w}\left(s_{1}\right) & \text { if }\left(F_{A}\right) \geq 0, \\ f_{w}\left(s_{2}\right) & \text { if }\left(F_{A}\right)<0 .\end{cases}
$$

where $F_{A}$ is defined above in section 4

In this section, we present numerical simulations that demonstrate the above method applied to problems with capillary heterogeneity and discontinuity. The boundary conditions for all cases involve specification of in-flow flux and constant saturation on the left hand boundary, pressure on the right hand out-flow boundary and solid wall zero normal flux on the top and bottom walls of the domains. First, we present a discontinuity. We also present test cases with discontinuous capillarity and full-tensor permeability fields and different residual oil saturations in two different rocks. We include a case using a centred approximation for capillary flux oil-phase mobility which leads to numerical instability and contrast to the stable results obtained using an upwind oil-phase mobility approximation based on capillary flux. The CFL number used

\subsection{Continuous capillarity}

We present a simulation of the displacement of oil by water from left to right in a layered channel. The domain of size $[2 \mathrm{~m} \times 0.9 \mathrm{~m}]$, consists of two alternating rock-types with different permeability values. The permeability of the domain is isotropic everywhere with $\boldsymbol{k}=k \boldsymbol{I} m D$. The middle rock is of low permeability $(k=0.01)$ compared to rest of the domain $(k=1.0)$, as shown in Fig. 3a. Quadratic relative permeabilities are chosen for the whole domain in this test case. A triangular grid is used for this test case and subsequent cases. The capillary-saturation relationship is defined by

$$
\phi_{c}=-\frac{\varphi}{\sqrt{k}} \ln s
$$

which varies for a heterogeneous permeable domain. The capillary pressure is continuous with constant threshold pressure thoughout the whole domain. The saturation fields at PVI $=0.125$ and $\mathrm{PVI}=0.25$ are shown in Fig. $3 \mathrm{~b}$ and Fig. $3 \mathrm{c}$ respectively, which show effects of heterogeneity of the domain on oil displacement, consistent with the literature (Friis and Evje, 2012). 


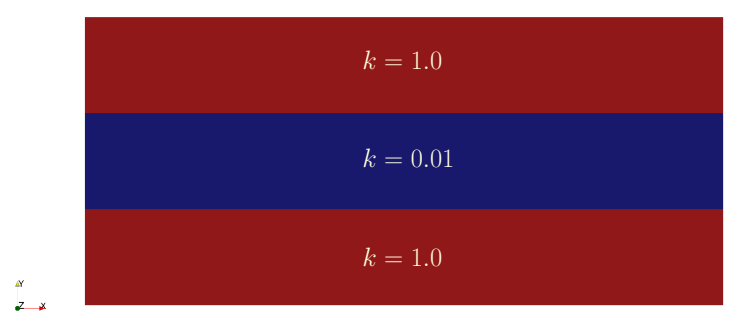

(a) Rocks with heterogeneity

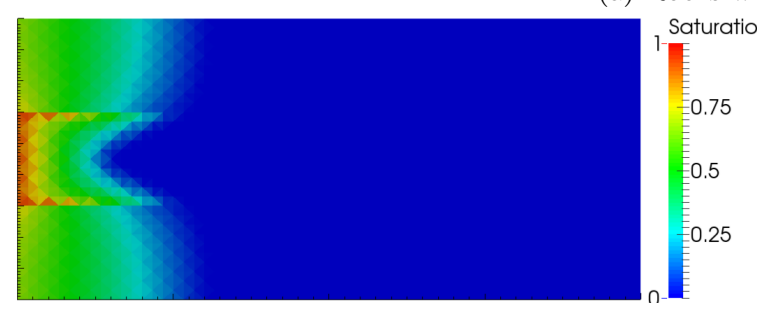

(b) Saturation plot at PVI=0.125.

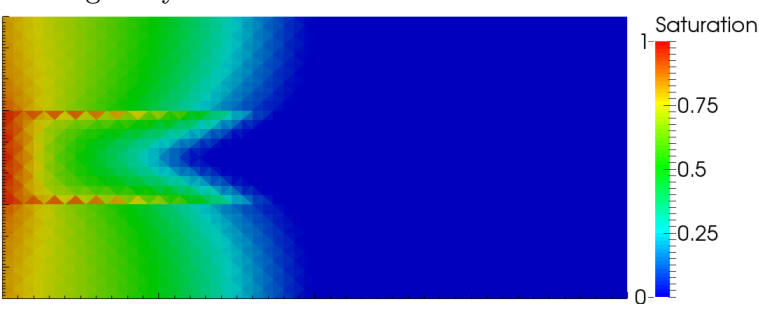

(c) Saturation plot at PVI $=0.25$.

Fig. 3: Channel flow in a domain consisting of two rock-types with equal threshold capillary pressure values.

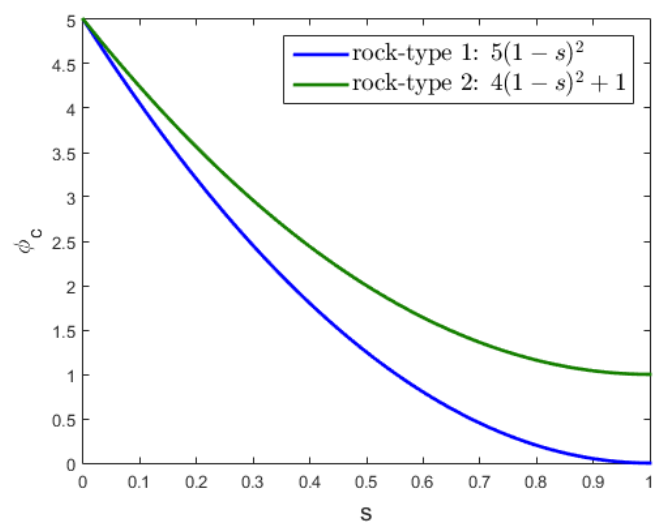

Fig. 4: Capillary pressure functions with different threshold pressures.

\subsection{Discontinuous capillarity}

Now, we present simulations where the capillary pressure can be discontinuous across the interface between two different rock-types. Consider the capillary-saturation curves, as shown in Fig. 4, which are defined by:

$$
\begin{array}{ll}
\text { rock-type 1: } & \phi_{c}=5(1-\hat{s})^{2} \\
\text { rock-type 2: } & \phi_{c}=4(1-\hat{s})^{2}+1
\end{array}
$$

where, $\hat{s}$ is the normalised variable defined by

$$
\hat{s}=\frac{s-s_{w c}}{1-s_{o r}-s_{w c}}
$$

where $s_{o r}=s_{w c}=0.0$ so that $s=\hat{s}$ inside the whole domain for this section. It is evident that there is a difference in the threshold pressures of the two rock-types and the capillary pressure is discontinuous whenever capillary pressure in the rock-type 1 is less than the threshold pressure of rock-type 2. Two cases are considered: Case (A) where the rock in the inner rectangle of the domain has rock-type 2 (Eq. (13)) and the rest of the domain has rock-type 1 (Eq. (12)), as shown in Fig. 5a. Case (B) is the opposite of case (A), where now the rock in the inner rectangle of domain has rock-type 1 (Eq. (12)) and the rest of the domain has rock-type 2 (Eq. (13)), as shown in Fig. 5b. The relative permeability functions for the whole domain are given by

$$
k_{r w}=\hat{s}^{\frac{2+3 \theta}{\theta}}, \quad k_{r o}=(1-\hat{s})^{2}\left(1-\hat{s}^{\frac{2+\theta}{\theta}}\right)
$$




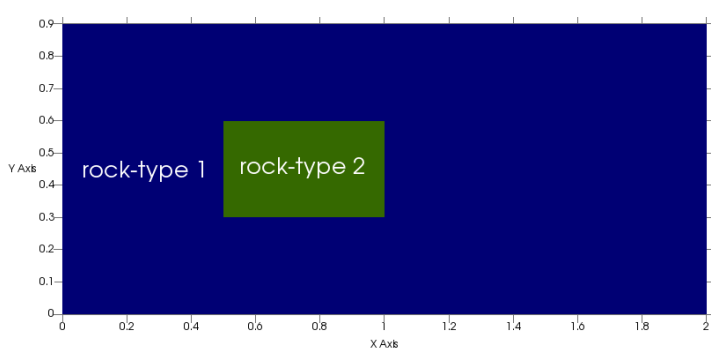

(a) Case A: Rocks with capillary heterogeneity (Constant permeability)

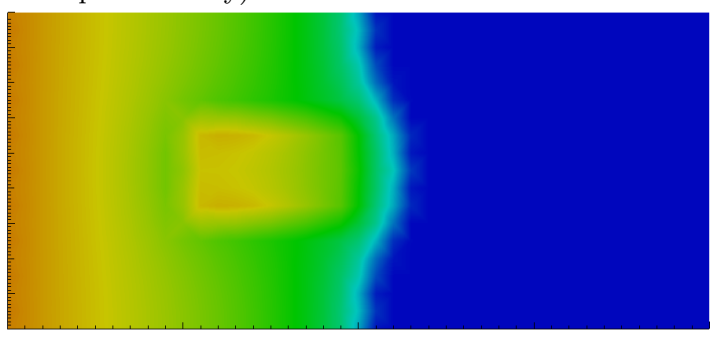

(c) Case A: Saturation plot at PVI=0.5

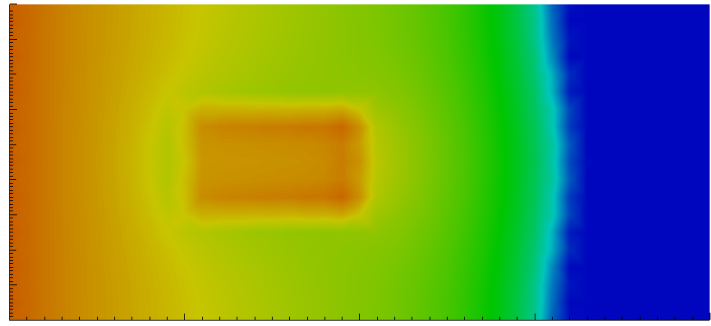

(e) Case A: Saturation plot at PVI $=0.75$

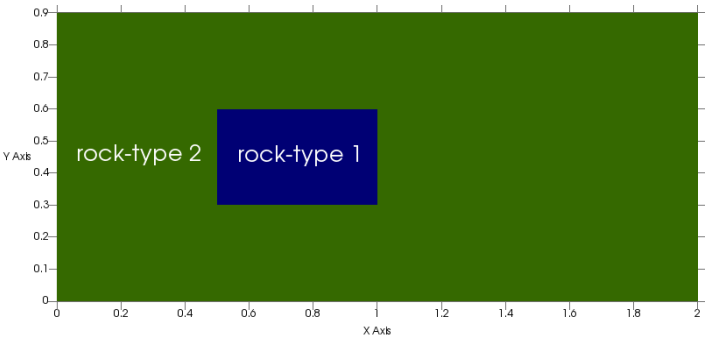

(b) Case B: Rocks with capillary heterogeneity (Constant permeability)

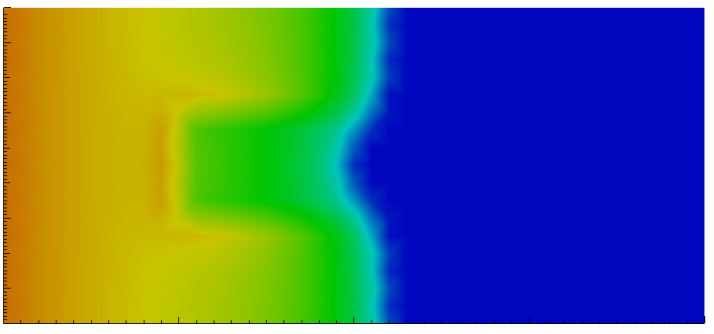

(d) Case B: Saturation plot at PVI=0.5

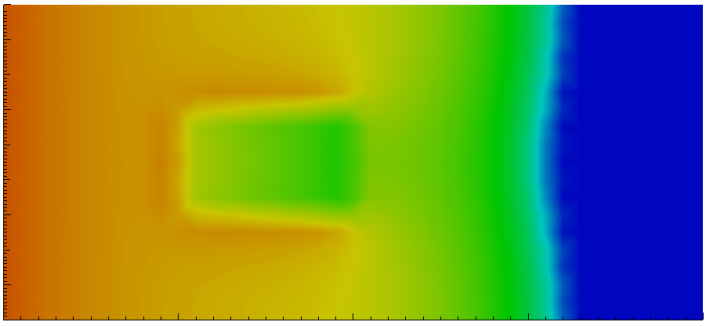

(f) Case B: Saturation plot at PVI $=0.75$

Fig. 5: Simulation results for the cases of capillary discontinuity corresponding to capillary pressure curves shown in Fig. 4

where $\theta$ is the characteristic of the inhomogeneity of the medium and $\theta=2$ is used here. The domain size and grid used in this section are the same as in the previous section 6.1. Permeability values of both rocktypes are equal $(\boldsymbol{k}=\boldsymbol{I} m D)$. Figures $5 \mathrm{c}$ and $5 \mathrm{~d}$ show the saturation plots for cases $(\mathrm{A})$ and $(\mathrm{B})$ respectively at $\mathrm{PVI}=0.5$. Saturation fields for both cases at PVI $=0.75$ are shown in Figs. $5 \mathrm{e}$. $5 \mathrm{f}$. As a consequence, in case (A) the non-wetting phase flows out of the rectangle while being replaced by the wetting phase, as the outer domain has lower entry pressure. Conversely in case (B) the non-wetting phase is trapped due to the higher entry pressure of the outer domain, a phenomenon known as oil trapping in the literature (e.g., van Duijn et al, 1995, Brenner et al, 2013).

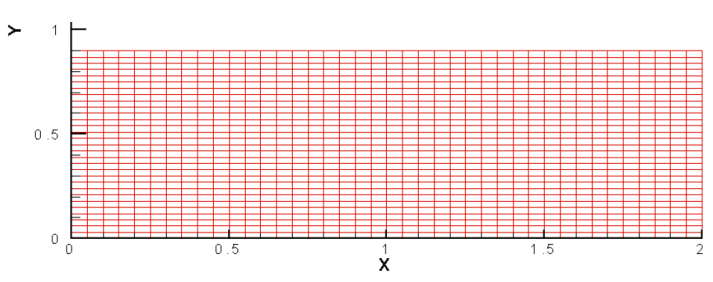

(a) Quadrilateral mesh, 1200 cells

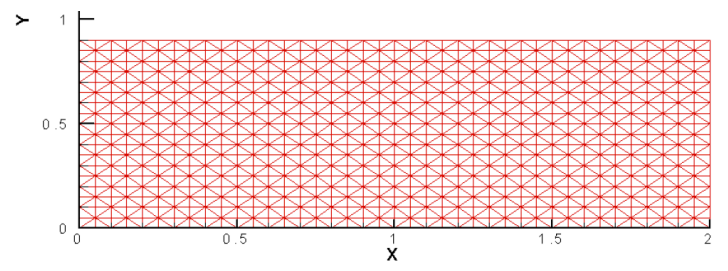

(b) Triangular mesh, 1440 cells

Fig. 6: Meshes used for discontinuous capillarity case with full-tensor anisotropic permeability field

We now present simulation results for a test case with a full-tensor permeability field i.e. $\boldsymbol{k}=\left(\begin{array}{c}7.750 \\ 3.897 \\ 3.250\end{array}\right) m D$ (anisotropy ratio of $10: 1$ at $30^{\circ}$ to the horizontal reference axis). The domain, capillary heterogeneity and 


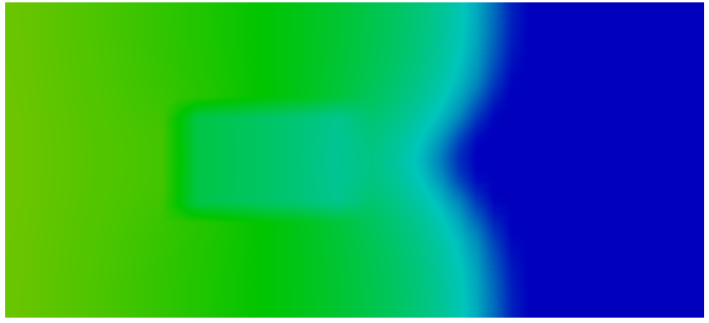

(a) Quadrilateral mesh, TPFA

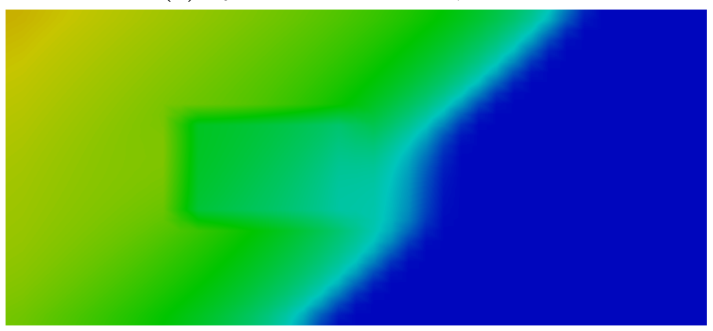

(c) Quadrilateral mesh, CVD-MPFA

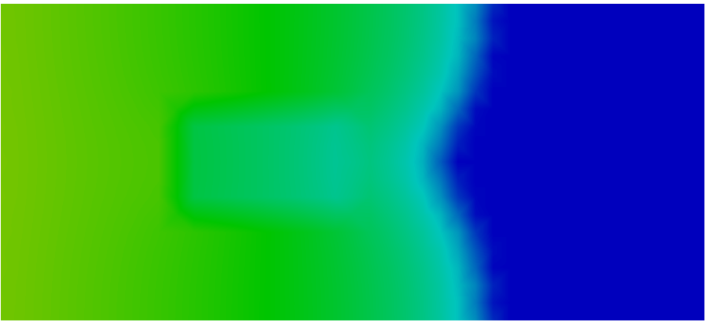

(b) Triangular mesh, TPFA

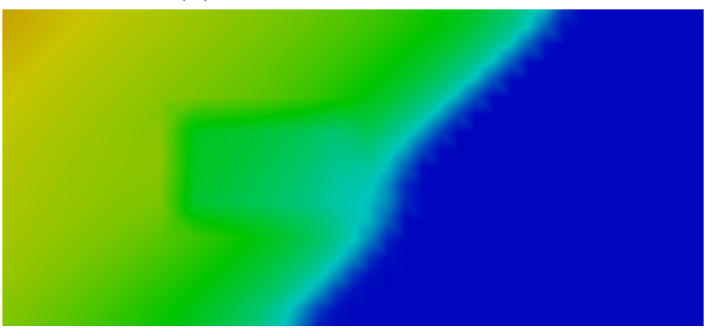

(d) Triangular mesh, CVD-MPFA

Fig. 7: Saturation results at PVI $=0.5$ for the case of capillary discontinuity with full-tensor permeability field

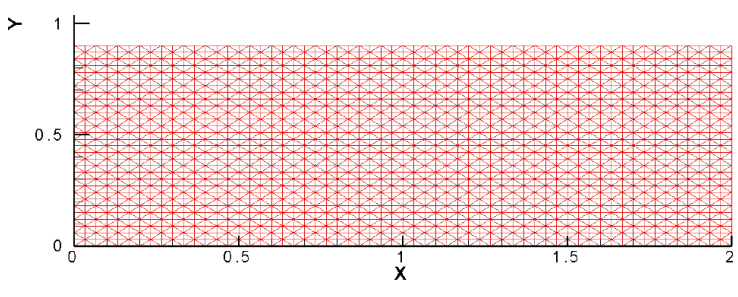

(a) Fine triangular mesh, 3600 cells

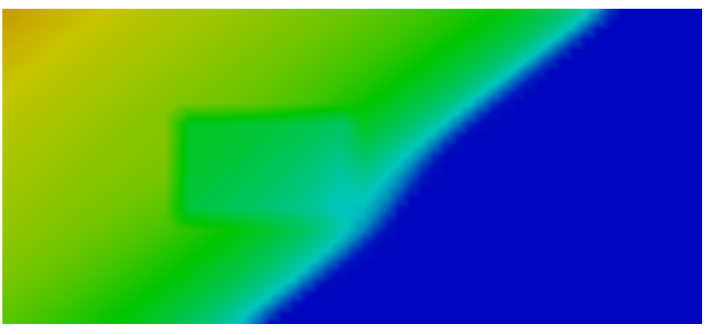

(b) Saturation plot using fine triangular mesh

Fig. 8: Fine scale saturation result at PVI=0.5 via CVD-MPFA for the case of capillary discontinuity with full-tensor permeability field

boundary conditions are the same as Case (B) of the previous section 6.2. Results are computed on two mesh types; the first is a quadrilateral mesh and the second is a triangular mesh, as shown in Fig. 6. Solutions computed using both the new CVD-MPFA method and the conventional two-point flux approximation (TPFA) method on both mesh types. The simulation results at PVI $=0.5$ and $\mathrm{PVI}=0.75$ are presented in Figs. 7 and 9. The saturation fields computed by the CVD-MPFA method clearly show that the new method captures the influence of full-tensor anisotropy effects on the flow field while resolving oil trapping. In contrast the results obtained via TPFA do not account for a full-tensor permeability field and clearly illustrate the $O(1)$ error in the solution, where only diagonal tensor effects can be seen. The saturation fields computed on both mesh types by the CVD-MPFA method are in good agreement with no indication of grid orientation effects. For reference the results computed by the new CVD-MPFA method on a fine triangular mesh are presented in Figs. $8 \mathrm{~b}$ and $9 \mathrm{e}$, which verifies the consistent trends in anisotropic flow field behaviour predicted by the CVD-MPFA method on coarser grids for both grid types.

\subsection{Different residual oil saturations}

In this section, we consider different residual saturations in the two rock-types. The domain, capillary heterogeneity and boundary conditions are the same as Case (B) of the section 6.2 where, capillary functions are defined by Eqs. (12) and (13) and relative permeability functions are defined by Eq. (15). Here $s_{\text {or }}=0.1$, $s_{w c}=0.0$ inside the inner rock-type 1 , and $s_{o r}=s_{w c}=0.0$ elsewhere. The capillary-saturation curves are shown in Fig. 10. Results are computed for domains with isotropic as well as full-tensor permeability fields as defined above, on both coarse and fine triangular meshes as shown in Fig. 11. The solutions computed using CVD-MPFA for the domain with isotropic permeability field are shown in Fig. 12 while solutions computed using CVD-MPFA for the domain with a full-tensor permeability field are shown in Fig. 13. 


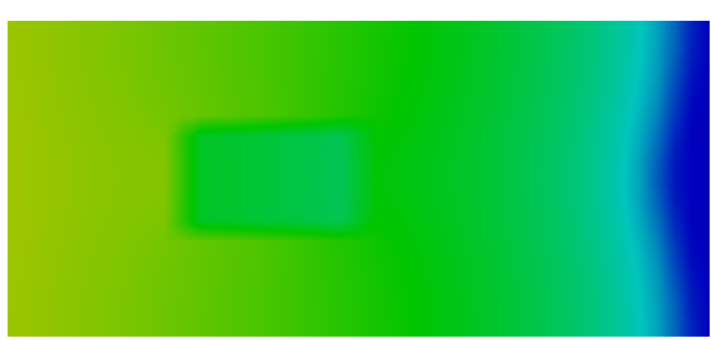

(a) Quadrilateral mesh, TPFA

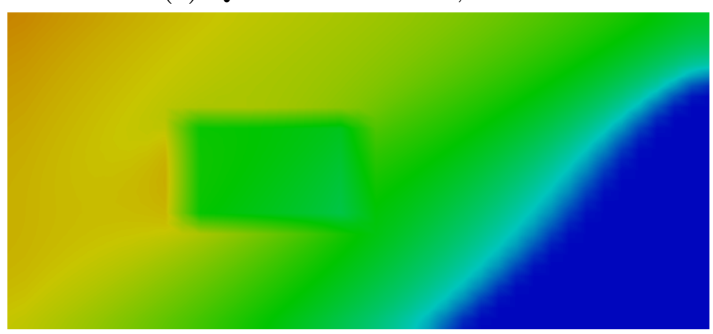

(c) Quadrilateral mesh, CVD-MPFA

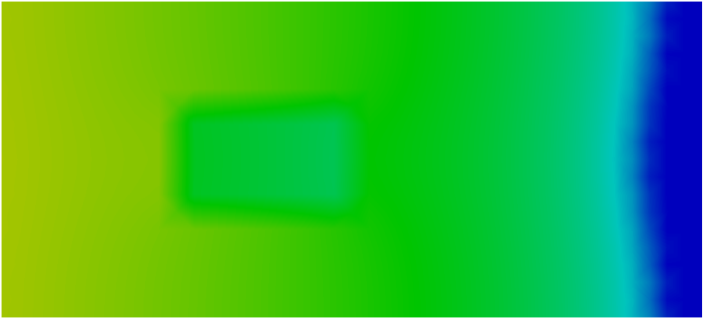

(b) Triangular mesh, TPFA

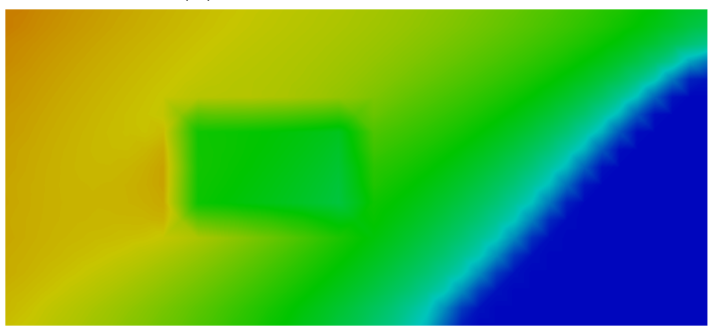

(d) Triangular mesh, CVD-MPFA

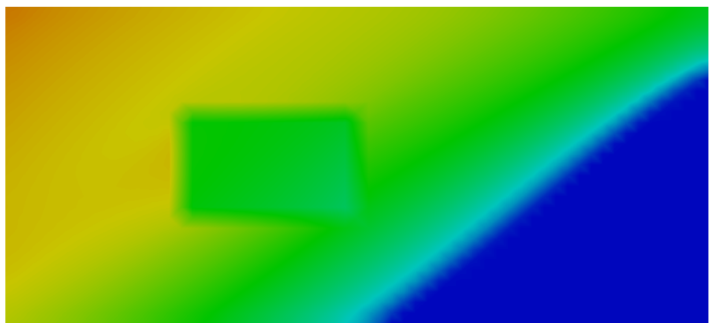

(e) Fine triangular mesh, CVD-MPFA

Fig. 9: Saturation results at PVI= 0.75 for the case of capillary discontinuity with full-tensor permeability field

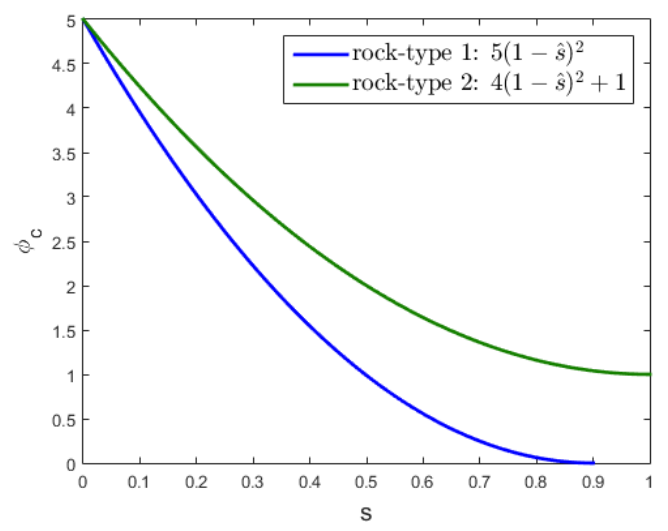

Fig. 10: Capillary pressure functions with different threshold pressures and $s_{\text {or }}=0.1$ inside the inner rock-type 1.

Results computed using TPFA for the domain with a full-tensor permeability field are shown in Fig. 14 which again fail to capture full-tensor permeability effects due to the $O(1)$ error in the TPFA flux. The TPFA results are in sharp contrast to those of the consistent CVD-MPFA scheme c.f. Fig. 13 which capture the flow effects induced by the full-tensor permeability field.

6.5 Centred capillary flux phase mobility gives instability

The results presented in Fig. 15 are for a diagonal tensor with $s_{\text {or }}=0$ and computed on the triangular mesh

Fig. $6 \mathrm{~b}$ with the $V a$ scheme using a centred approximation for capillary flux oil-phase mobility $\lambda_{o}$. Local 


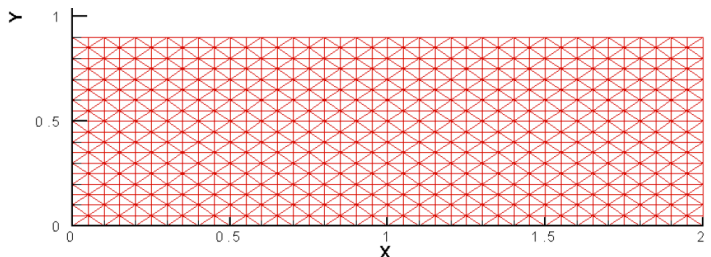

(a) Mesh 1, 1440 cells

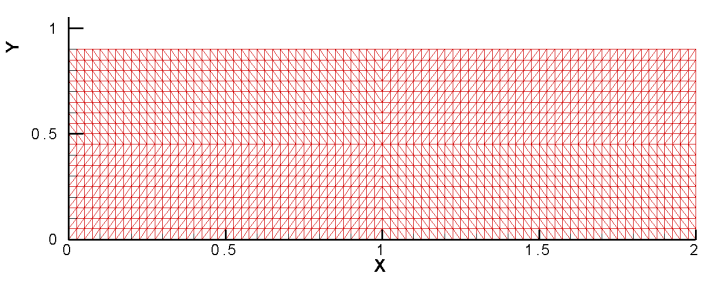

(b) Mesh 2, 2880 cells

Fig. 11: Meshes used for discontinuous capillarity case and non-zero residual oil saturation $\left(s_{\text {or }}=0.1\right)$ in inner rock

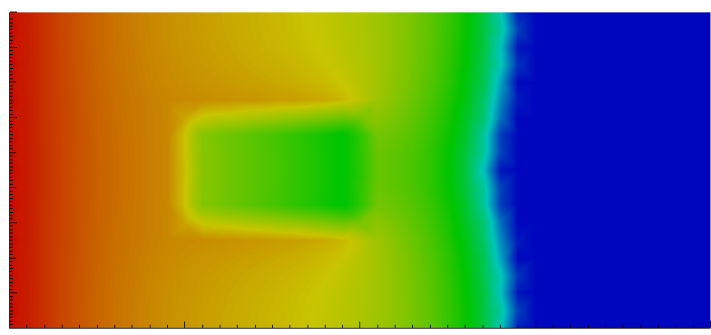

(a) Mesh 1, PVI=0.5

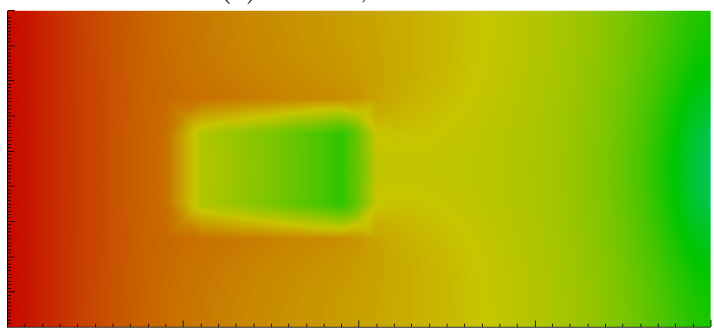

(c) Mesh 1, PVI $=0.75$

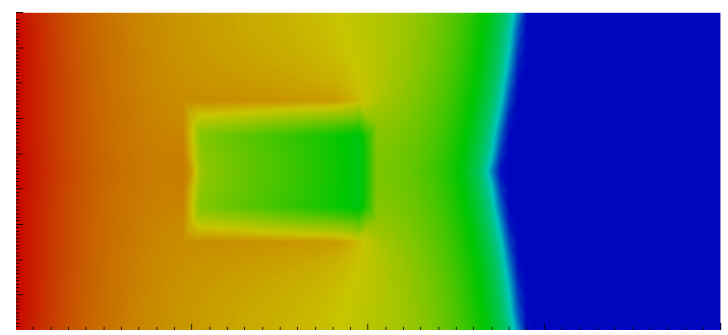

(b) Mesh 2, PVI $=0.5$

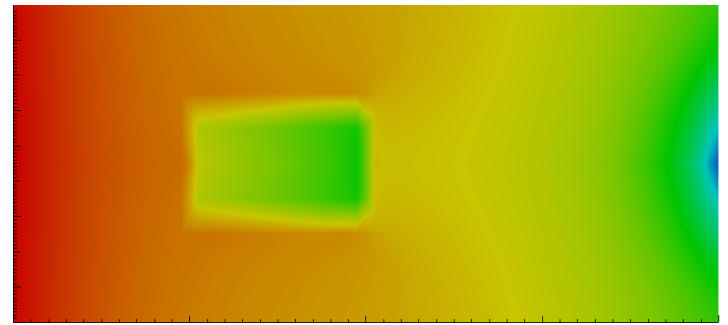

(d) Mesh 2, PVI= 0.75

Fig. 12: Saturation results at $\mathrm{PVI}=0.5$ and $\mathrm{PVI}=0.75$ via CVD-MPFA for the case of capillary discontinuity with isotropic permeability field and $s_{\text {or }}=0.1$ in inner rock

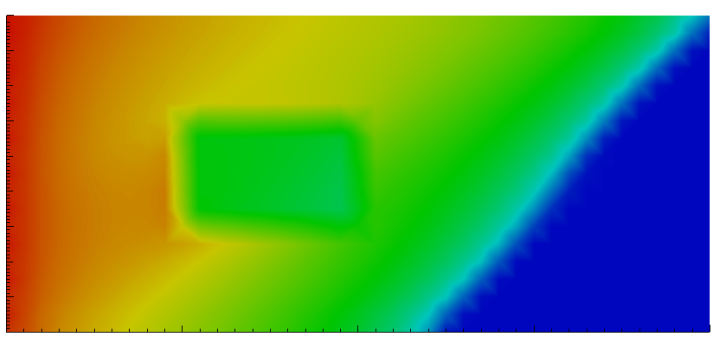

(a) Mesh 1, PVI $=0.5$

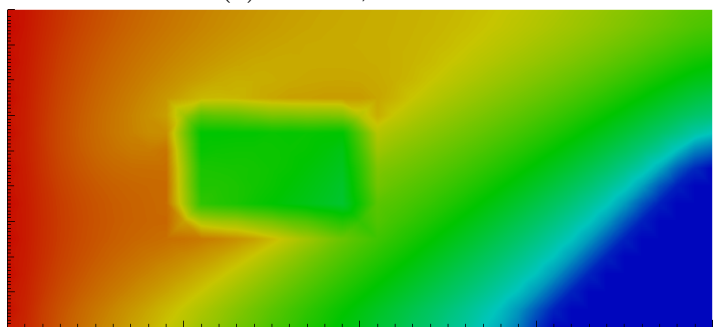

(c) Mesh 1, PVI $=0.75$

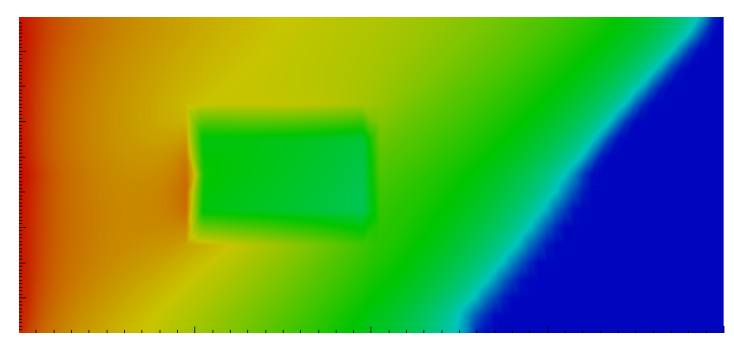

(b) Mesh 2, PVI $=0.5$

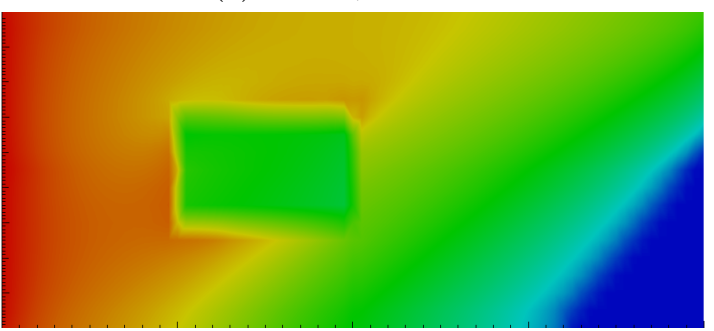

(d) Mesh 2, PVI $=0.75$

Fig. 13: Saturation results at $\mathrm{PVI}=0.5$ and $\mathrm{PVI}=0.75$ via CVD-MPFA for the case of capillary discontinuity with full-tensor permeability field and $s_{\text {or }}=0.1$ in inner rock

instability can be seen adjacent to the left hand side of the inner rectangular boundary in Fig. 15(a) which then spreads around the upper and lower perimeter in Fig. 15(b). However in the work presented in this paper we employ an upwind approximation for capillary flux oil-phase mobility $\lambda_{o}$ according to the sign of 


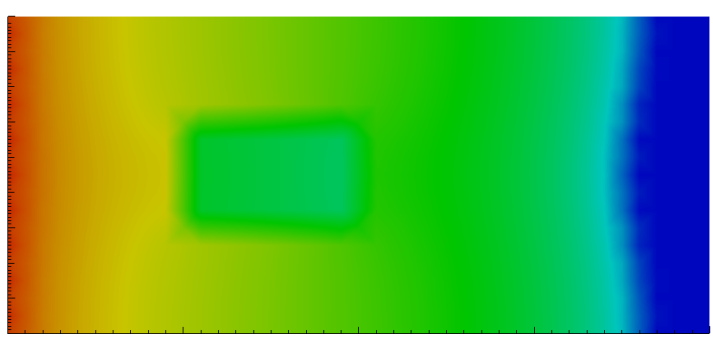

(a) $\mathrm{PVI}=0.5$

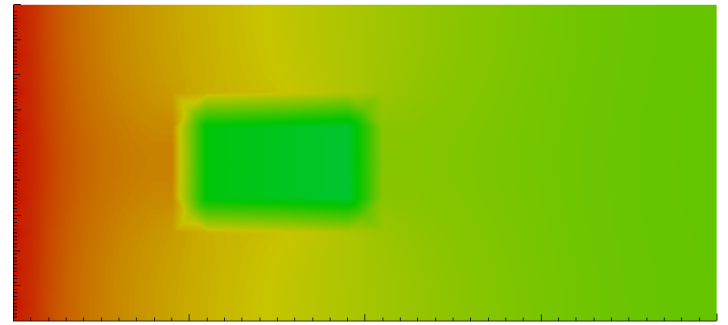

(b) $\mathrm{PVI}=0.75$

Fig. 14: Saturation results at PVI=0.5 and PVI=0.75 via TPFA using Mesh 1 (Fig. 11a) for the case of capillary discontinuity with full-tensor permeability field and $s_{\text {or }}=0.1$ in inner rock

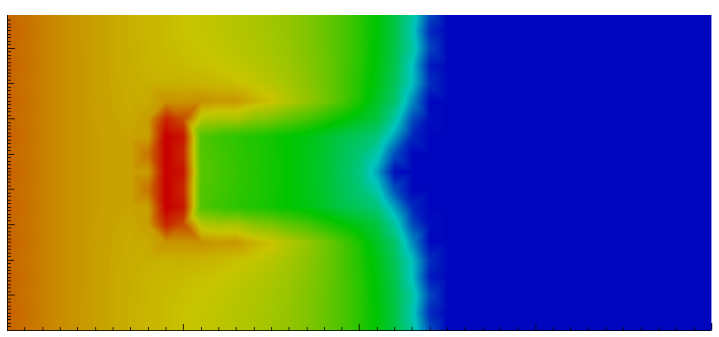

(a) $\mathrm{PVI}=0.5$

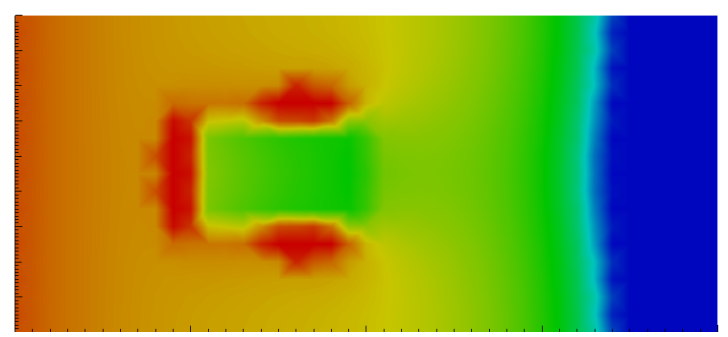

(b) $\mathrm{PVI}=0.75$

Fig. 15: Simulation results for the case of capillary discontinuity corresponding to capillary pressure curves shown in Fig. 4 and case B described in section 6.2 with isotropic permeability field and computed on mesh Fig. 6b using a centred approximation for phase mobility

the capillary pressure flux, which aids stability, together with the upwind $V a$ flux and CFL condition based on the $\mathrm{Va}$ flux. The specific example results of Fig. $15(\mathrm{a}, \mathrm{b})$ should be contrasted with the corresponding stable results shown above in Figs. $5 \mathrm{~d}$ and $5 \mathrm{f}$ respectively, for the same case, computed on the same mesh but instead using the upwind oil-phase mobility approximation.

\section{Conclusions}

We have presented a new cell-centred finite-volume formulation for two-phase flow where capillary pressure can be discontinuous across the interfaces of heterogeneous regions in the domain. The CVD-MPFA scheme (Friis et al, 2008), designed for the solution of the pressure equation with discontinuous full-tensor permeability coefficients, is further developed here and leads to a new CVD-MPFA capillary pressure operator. The new method is specifically formulated to handle discontinuous capillary pressure. Simulations are presented which include cases of oil trapping and demonstrate the ability of the method to resolve discontinuous capillary pressure fields in the presence of both diagonal and full-tensor permeability fields. In contrast to the standard TPFA method which is unable to resolve full-tensor flow fields, consistent results are computed by the CVD-MPFA method on both structured and unstructured grids that are free of grid orientation, and demonstrate the ability of the method to resolve anisotropy induced flow fields. In addition to an upwind approximation for the saturation equation $V a$ flux and the associated stability limit, the importance of upwinding oil-phase mobility according to capillary flux is demonstrated for discontinuous capillary pressure, and leads to a hybrid upwind $V a$ formulation constructed within a novel CVD-MPFA framework. 


\section{Nomenclature}

\begin{tabular}{ll|ll}
\multicolumn{2}{c}{ CVD-MPFA } & control-volume distributed multi-point flux approximation \\
$\phi$ & pressure & $F$ & flux \\
$\Phi$ & vector of pressures & $\boldsymbol{k}$ & permeability tensor \\
$\varphi$ & porosity & $\boldsymbol{K}$ & $\frac{\boldsymbol{k}}{\mu}$ \\
$\lambda_{w}$ & water mobility & $q$ & quadrature of CVD-MPFA schemes \\
$\lambda_{o}$ & oil mobility & $q_{w}$ & known water phase source term \\
$\Lambda$ & total mobility $\lambda_{w}+\lambda_{o}$ & $q_{o}$ & known oil phase source term \\
$\mu$ & viscosity & $s$ & saturation \\
& & $\boldsymbol{v}$ & velocity
\end{tabular}

$\begin{array}{ll}c & \text { capillary } \\ o & \text { oil } \\ w & \text { water }\end{array}$

Superscript

$t r$

transpose

\section{References}

Arbogast T, Juntunen M, Pool J, Wheeler M: A discontinuous galerkin method for two-phase flow in a porous medium enforcing h (div) velocityand continuous capillary pressure. Comput. Geosci. 17(6):10551078 (2013)

Aziz K, Settari A: Petroleum Reservoir Simulation. Elsevier Applied Science Publishers (1986)

Bastian P: A fully-coupled discontinuous Galerkin method for two-phase flow in porous media with discontinuous capillary pressure. Comput. Geosci. 18(5):779-796 (2014)

Brenner K, Cancès C, Hilhorst D: Finite volume approximation for an immiscible two-phase flow in porous media with discontinuous capillary pressure. Comput. Geosci. 17(3):573-597 (2013)

Brenner K, Hennicker J, Masson R, Samier P: Gradient discretization of hybrid-dimensional Darcy flow in fractured porous media with discontinuous pressures at matrix-fracture interfaces. IMA J. Numer. Anal. $37(3): 1551-1585$ (2016)

Cancès C: Finite volume scheme for two-phase flows in heterogeneous porous media involving capillary pressure discontinuities. ESAIM: Math. Model. Numer. Anal. 43(5):973-1001 (2009)

van Duijn CJ, de Neef MJ: Similarity solution for capillary redistribution of two phase in a porous medium with a single discontinuity. Adv. Water Resour. 21:451-461 (1998)

van Duijn CJ, Molenaar J, de Neef MJ: The effect of capillary forces on immiscible two-phase flow in heterogeneous porous media. Transp. Porous Media 21:71-93 (1995)

Edwards MG, Rogers CF: Finite volume discretization with imposed flux continuity for the general tensor pressure equation. Comput. Geosci. 02(04):259-290 (1998)

Enchéry G, Eymard R, Michel A: Numerical approximation of a two-phase flow problem in a porous medium with discontinuous capillary forces. SIAM J. Numer. Anal. 43(6):2402-2422 (2006)

Friis HA, Evje S: Numerical treatment of two-phase flow in capillary heterogeneous porous media by finitevolume approximations. Int. J. Numer. Anal. Mod. 9(3):505-528 (2012)

Friis HA, Edwards MG, Mykkeltveit J: Symmetric positive definite flux-continuous full-tensor finite-volume schemes on unstructured cell-centered triangular grids. SIAM J. Sci. Comput. 31(02):1192-1220 (2008)

Hamon F, Mallison BT, Tchelepi HA: Implicit hybrid upwinding for two-phase flow in heterogeneous porous media with buoyancy and capillarity. Comput. Meth. Appl. Mech. Eng. 331:701-727 (2018)

Helmig R, Huber R: Comparison of Galerkin-type discretization techniques for two-phase flow in heterogeneous porous media. Adv. Water Resour. 21(8):697-711 (1998)

Hoteit H, Firoozabadi A: Numerical modeling of two-phase flow in heterogeneous permeable media with different capillarity pressures. Adv. Water Resour. 31:56-73 (2008)

LeVeque RJ: Numerical Methods for Conservation Laws. Birkhäuser Verlag (1992)

Li B, Tchelepi HA: Nonlinear analysis of multiphase transport in porous media in the presence of viscous, buoyancy, and capillary forces. J. Comput. Phys. 297:104-131 (2015) 
Moortgat J, Firoozabadi A: Three-phase compositional modeling with capillarity in heterogeneous and fractured media. SPE J. 18(06):1-150 (2013)

Niessner J, Helmig R, Jakobs H, Roberts J: Interface condition and linearization schemes in the newton iterations for two-phase flow in heterogeneous porous media. Adv. Water Resour. 28(7):671-687 (2005)

Papafotiou A, Sheta H, Helmig R: Numerical modeling of two-phase hysteresis combined with an interface condition for heterogeneous porous media. Comput. Geosci. 14(2):273-287 (2010)

330 Wolff M, Cao Y, Flemisch B, Helmig R, Wohlmuth B: Multi-point flux approximation L-method in 3D: Numerical convergence and application to two-phase flow through porous media. In: Simul. Flow Porous Media, pp 39-80. de Gruyter (2013)

Xie Y, Edwards MG: Higher resolution total velocity Vt and Va finite-volume formulations on cell-centred structured and unstructured grids. Comput. Geosci. 21(5):921-936 (2017) 